\title{
On the Intersection Equation of a Hyperboloid and a Plane
}

\author{
Peter Paul Klein \\ Clausthal University of Technology, Clausthal-Zellerfeld, Germany \\ Email: klein@rz.tu-clausthal.de
}

Received October 25, 2013; revised November 25, 2013; accepted December 2, 2013

Copyright (C) 2013 Peter Paul Klein. This is an open access article distributed under the Creative Commons Attribution License, which permits unrestricted use, distribution, and reproduction in any medium, provided the original work is properly cited. In accordance of the Creative Commons Attribution License all Copyrights (C) 2013 are reserved for SCIRP and the owner of the intellectual property Peter Paul Klein. All Copyright (C) 2013 are guarded by law and by SCIRP as a guardian.

\begin{abstract}
In this note, the ideas employed in [1] to treat the problem of an ellipsoid intersected by a plane are applied to the analogous problem of a hyperboloid being intersected by a plane. The curves of intersection resulting in this case are not only ellipses but rather all types of conics: ellipses, hyperbolas and parabolas. In text books of mathematics usually only cases are treated, where the planes of intersection are parallel to the coordinate planes. Here the general case is illustrated with intersecting planes which are not necessarily parallel to the coordinate planes.
\end{abstract}

Keywords: Hyperboloid; Intersection Equation of Hyperboloid and Plane

\section{Introduction}

The problem of a hyperboloid being intersected by a plane is described in Section 1. The means to treat the problem are provided in Sections 2, 3 and 4. In the end of Section 4 first results can be formulated in Corollaries 3 and 4. Further results concerning the center of the conic of intersection are given in Section 5. Finally in Section 6 the case of a parabola as intersecting curve is treated.

Let a hyperboloid be given with the three positive semi axes $a, b, c$

$$
\frac{x_{1}^{2}}{a^{2}}+\frac{x_{2}^{2}}{b^{2}}-\frac{x_{3}^{2}}{c^{2}}= \pm 1
$$

where +1 on the right hand side of (1) corresponds to a hyperboloid of one sheet, -1 on the right hand side of (1) to a hyperboloid of two sheets. Let furthermore a plane be given with the unit normal vector

$$
\boldsymbol{n}=\left(n_{1}, n_{2}, n_{3}\right)^{\mathrm{T}},
$$

which contains an interior point or a boundary point $\boldsymbol{q}=\left(q_{1}, q_{2}, q_{3}\right)^{\mathrm{T}}$ of hyperboloid (1). A plane spanned by vectors $\boldsymbol{r}=\left(r_{1}, r_{2}, r_{3}\right)^{\mathrm{T}}, \quad \boldsymbol{s}=\left(s_{1}, s_{2}, s_{3}\right)^{\mathrm{T}}$ and containing the point $\boldsymbol{q}$ is described in parametric form by

$$
\boldsymbol{x}=\boldsymbol{q}+\boldsymbol{t r}+u \boldsymbol{s} \text { with } \boldsymbol{x}=\left(x_{1}, x_{2}, x_{3}\right)^{\mathrm{T}} .
$$

Inserting the components of $x$ into the Equation of hyperboloid (1) leads to the line of intersection as a quadratic form in the variables $t$ and $u$. Let the scalar product in $\boldsymbol{R}^{3}$ for two vectors $\boldsymbol{v}=\left(v_{1}, v_{2}, v_{3}\right)^{\mathrm{T}}$ and $\boldsymbol{w}=\left(w_{1}, w_{2}, w_{3}\right)^{T}$ be denoted by

$$
(\boldsymbol{v}, \boldsymbol{w})=v_{1} w_{1}+v_{2} w_{2}+v_{3} w_{3} .
$$

With the diagonal matrices

$$
D_{ \pm}=\operatorname{diag}\left(\frac{1}{a}, \frac{1}{b}, \pm \frac{1}{c}\right)
$$

the line of intersection has the form:

$$
\begin{aligned}
& (t, u)\left(\begin{array}{ll}
\left(D_{+} \boldsymbol{r}, D_{-} \boldsymbol{r}\right) & \left(D_{+} \boldsymbol{r}, D_{-} \boldsymbol{s}\right) \\
\left(D_{+} \boldsymbol{r}, D_{-} \boldsymbol{s}\right) & \left(D_{+} \boldsymbol{s}, D_{-} \boldsymbol{s}\right)
\end{array}\right)\left(\begin{array}{l}
t \\
u
\end{array}\right) \\
& +2\left(\left(D_{+} \boldsymbol{q}, D_{-} \boldsymbol{r}\right),\left(D_{+} \boldsymbol{q}, D_{-} \boldsymbol{s}\right)\right)\left(\begin{array}{l}
t \\
u
\end{array}\right) \\
& = \pm 1-\left(D_{+} \boldsymbol{q}, D_{-} \boldsymbol{q}\right) .
\end{aligned}
$$

As $\boldsymbol{q}$ is an interior point or a boundary point of hyperboloid (1) the right-hand side of Equation (3) is nonnegative. Since $\left(D_{+} \boldsymbol{x}, D_{-} \boldsymbol{y}\right)$ need not be a scalar product in $\boldsymbol{R}^{3}$, the $2 \times 2$ matrix in Equation (3) is in general no Gram matrix. If the $2 \times 2$ matrix in (3) is positive definite, then the line of intersection is an ellipse.

Let $\boldsymbol{r}$ and $\boldsymbol{s}$ be unit vectors orthogonal to the unit normal vector $\boldsymbol{n}$ of plane (2)

$$
\begin{aligned}
& (\boldsymbol{r}, \boldsymbol{r})=r_{1}^{2}+r_{2}^{2}+r_{3}^{2}=1, \\
& (\boldsymbol{n}, \boldsymbol{r})=n_{1} r_{1}+n_{2} r_{2}+n_{3} r_{3}=0,
\end{aligned}
$$




$$
\begin{aligned}
& (\boldsymbol{s}, \boldsymbol{s})=s_{1}^{2}+s_{2}^{2}+s_{3}^{2}=1, \\
& (\boldsymbol{n}, \boldsymbol{s})=n_{1} s_{1}+n_{2} s_{2}+n_{3} s_{3}=0,
\end{aligned}
$$

and orthogonal to eachother

$$
(\boldsymbol{r}, \boldsymbol{s})=r_{1} s_{1}+r_{2} s_{2}+r_{3} s_{3}=0 \text {. }
$$

Furthermore vectors $\boldsymbol{r}$ and $\boldsymbol{s}$ may be chosen such that

$$
\left(D_{+} \boldsymbol{r}, D_{-} \boldsymbol{s}\right)=\frac{r_{1} S_{1}}{a^{2}}+\frac{r_{2} S_{2}}{b^{2}}-\frac{r_{3} S_{3}}{c^{2}}=0
$$

holds. This will be shown in the next Section. Condition (7) ensures that the $2 \times 2$ matrix in (3) has diagonal form.

In case $\left(D_{+} \boldsymbol{r}, D_{-} \boldsymbol{r}\right) \neq 0$ and $\left(D_{+} \boldsymbol{s}, D_{-} \boldsymbol{s}\right) \neq 0$ the line of intersection reduces to

$$
\begin{aligned}
& \left(D_{+} \boldsymbol{r}, D_{-} \boldsymbol{r}\right)\left(t-t_{0}\right)^{2}+\left(D_{+} \boldsymbol{s}, D_{-} \boldsymbol{s}\right)\left(u-u_{0}\right)^{2} \\
& = \pm 1-d
\end{aligned}
$$

with

$$
t_{0}=-\frac{\left(D_{+} \boldsymbol{q}, D_{-} \boldsymbol{r}\right)}{\left(D_{+} \boldsymbol{r}, D_{-} \boldsymbol{r}\right)}, \quad u_{0}=-\frac{\left(D_{+} \boldsymbol{q}, D_{-} \boldsymbol{s}\right)}{\left(D_{+} \boldsymbol{s}, D_{-} \boldsymbol{s}\right)}
$$

and

$$
d=\left(D_{+} \boldsymbol{q}, D_{-} \boldsymbol{q}\right)-\frac{\left(D_{+} \boldsymbol{q}, D_{-} \boldsymbol{r}\right)^{2}}{\left(D_{+} \boldsymbol{r}, D_{-} \boldsymbol{r}\right)}-\frac{\left(D_{+} \boldsymbol{q}, D_{-} \boldsymbol{s}\right)^{2}}{\left(D_{+} \boldsymbol{s}, D_{-} \boldsymbol{s}\right)} .
$$

In case $d \neq \pm 1$ Equation (8) can be written as a conic in translational form

$$
\frac{\left(t-t_{0}\right)^{2}}{\alpha_{1}}+\frac{\left(u-u_{0}\right)^{2}}{\alpha_{2}}=1
$$

in the variables $t$ and $u$ with

$$
\alpha_{1}=\frac{ \pm 1-d}{\left(D_{+} \boldsymbol{r}, D_{-} \boldsymbol{r}\right)}, \quad \alpha_{2}=\frac{ \pm 1-d}{\left(D_{+} \boldsymbol{s}, D_{-} \boldsymbol{s}\right)} .
$$

For $\left(D_{+} \boldsymbol{r}, D_{-} \boldsymbol{r}\right)=0$ and $\left(D_{+} \boldsymbol{s}, D_{-} \boldsymbol{s}\right) \neq 0$ the line of intersection is of the form

$$
2\left(D_{+} \boldsymbol{q}, D_{-} \boldsymbol{r}\right) t+\left(D_{+} \boldsymbol{s}, D_{-} \boldsymbol{s}\right)\left(u-u_{0}\right)^{2}= \pm 1-\tilde{d}
$$

with

$$
u_{0}=-\frac{\left(D_{+} \boldsymbol{q}, D_{-} \boldsymbol{s}\right)}{\left(D_{+} \boldsymbol{s}, D_{-} \boldsymbol{s}\right)} \text { and } \tilde{d}=\left(D_{+} \boldsymbol{q}, D_{-} \boldsymbol{q}\right)-\frac{\left(D_{+} \boldsymbol{q}, D_{-} \boldsymbol{s}\right)^{2}}{\left(D_{+} \boldsymbol{s}, D_{-} \boldsymbol{s}\right)} \text {. }
$$

If $\left(D_{+} \boldsymbol{q}, D_{-} \boldsymbol{r}\right) \neq 0$ holds, (13) represents a parabola in the variables $t$ and $u$. This will be discussed further in Section 6.

In order to show that the expression $d$ in (10) is independent of the choice of $\boldsymbol{q}$ this vector may be decomposed orthogonally with respect to $\boldsymbol{n}$ :

$$
\boldsymbol{q}=\kappa \boldsymbol{n}+\mu \boldsymbol{r}+\nu \boldsymbol{s} \text { with } \kappa=(\boldsymbol{q}, \boldsymbol{n})
$$

where $\kappa$ is the distance of plane (2) from the origin. Substituting $\boldsymbol{q}$ into (10) one obtains employing (4), (5), (6) and (7)

$$
d=\kappa^{2}\left(\left(D_{+} \boldsymbol{n}, D_{-} \boldsymbol{n}\right)-\frac{\left(D_{+} \boldsymbol{n}, D_{-} \boldsymbol{r}\right)^{2}}{\left(D_{+} \boldsymbol{r}, D_{-} \boldsymbol{r}\right)}-\frac{\left(D_{+} \boldsymbol{n}, D_{-} \boldsymbol{s}\right)^{2}}{\left(D_{+} \boldsymbol{s}, D_{-} \boldsymbol{s}\right)}\right) .
$$

The following rules of computation for the cross product in $\boldsymbol{R}^{3}$ ([2], p.147) will be applied repeatedly later on. For vectors $\boldsymbol{x}, \boldsymbol{y}, \boldsymbol{z}, \boldsymbol{w}$ of $\boldsymbol{R}^{3}$ the identity of Lagrange holds

$$
(x \times y, z \times w)=(x, z)(y, w)-(y, z)(x, w)
$$

and the Grassmann expansion theorem for the double cross product

$$
x \times(y \times z)=(x, z) y-(x, y) z .
$$

\section{Construction of Vectors $r$ and $s$}

Let $\boldsymbol{r}$ be a unit vector orthogonal to the unit normal vector $\boldsymbol{n}$ of the plane, so that Equations (4) hold. A suitable vector $\boldsymbol{s}$ is obtained as a cross product

$$
\boldsymbol{s}=\boldsymbol{n} \times \boldsymbol{r} .
$$

Then Equations (5) and (6) are fulfilled: $\boldsymbol{s}$ is a unit vector, as can be shown by the identity of Lagrange (16), utilising $(\boldsymbol{n}, \boldsymbol{n})=1,(\boldsymbol{r}, \boldsymbol{r})=1$ and $(\boldsymbol{n}, \boldsymbol{r})=0$ :

$$
(\boldsymbol{s}, \boldsymbol{s})=(\boldsymbol{n} \times \boldsymbol{r}, \boldsymbol{n} \times \boldsymbol{r})=(\boldsymbol{n}, \boldsymbol{n})(\boldsymbol{r}, \boldsymbol{r})-(\boldsymbol{n}, \boldsymbol{r})^{2}=1 .
$$

Furthermore one obtains according to the rules applying to the spar product:

$$
\begin{aligned}
& (\boldsymbol{n}, \boldsymbol{s})=(\boldsymbol{n}, \boldsymbol{n} \times \boldsymbol{r})=\operatorname{det}(\boldsymbol{n}, \boldsymbol{n}, \boldsymbol{r})=0, \\
& (\boldsymbol{r}, \boldsymbol{s})=(\boldsymbol{r}, \boldsymbol{n} \times \boldsymbol{r})=\operatorname{det}(\boldsymbol{r}, \boldsymbol{n}, \boldsymbol{r})=0 .
\end{aligned}
$$

In case Equation (7) is not fulfilled for the initially chosen vectors $\boldsymbol{r}$ and $\boldsymbol{s}$, i.e. $\left(D_{+} \boldsymbol{r}, D_{-} \boldsymbol{s}\right) \neq 0$, the following transformation may be performed with $\omega \in[-\pi, \pi)$

$$
\begin{aligned}
& \tilde{\boldsymbol{r}}=\cos \omega \boldsymbol{r}+\sin \omega \boldsymbol{s}, \\
& \tilde{\boldsymbol{s}}=-\sin \omega \boldsymbol{r}+\cos \omega \boldsymbol{s} .
\end{aligned}
$$

The transformed vectors $\tilde{\boldsymbol{r}}$ and $\tilde{\boldsymbol{s}}$ satisfy the following conditions: $(\tilde{\boldsymbol{r}}, \tilde{\boldsymbol{r}})=1, \quad(\boldsymbol{n}, \tilde{\boldsymbol{r}})=0$ and $\tilde{\boldsymbol{s}}=\boldsymbol{n} \times \tilde{\boldsymbol{r}}$, which imply conditions (4), (5) and (6). The expression

$$
\begin{aligned}
\left(D_{+} \tilde{\boldsymbol{r}}, D_{-} \tilde{\boldsymbol{s}}\right) & =\left(\left(D_{+} \boldsymbol{s}, D_{-} \boldsymbol{s}\right)-\left(D_{+} \boldsymbol{r}, D_{-} \boldsymbol{r}\right)\right) \frac{1}{2} \sin 2 \omega \\
& +\left(D_{+} \boldsymbol{r}, D_{-} \boldsymbol{s}\right) \cos 2 \omega
\end{aligned}
$$

becomes zero, when choosing $\omega$ such that

$$
\frac{\left(D_{+} \boldsymbol{r}, D_{-} \boldsymbol{r}\right)-\left(D_{+} \boldsymbol{s}, D_{-} \boldsymbol{s}\right)}{2\left(D_{+} \boldsymbol{r}, D_{-} \boldsymbol{s}\right)}=\cot 2 \omega
$$

holds.

Corollary 1: For the unit vectors $\boldsymbol{r}$ and $\boldsymbol{n}$ ortho- 
gonal to each other and $\boldsymbol{s}=\boldsymbol{n} \times \boldsymbol{r}$ the following statement holds:

$$
r_{i}^{2}+s_{i}^{2}+n_{i}^{2}=1 \text { for } i=1,2,3 .
$$

This statement follows by substituting the definition of $\boldsymbol{s}$ and utilising $(\boldsymbol{n}, \boldsymbol{n})=1,(\boldsymbol{r}, \boldsymbol{r})=1$ and $(\boldsymbol{n}, \boldsymbol{r})=0$. For $i=1$ one obtains for instance:

$$
\begin{aligned}
r_{1}^{2}+s_{1}^{2}+n_{1}^{2} & =r_{1}^{2}+\left(n_{2} r_{3}-r_{2} n_{3}\right)^{2}+n_{1}^{2} \\
& =r_{1}^{2}+n_{2}^{2} r_{3}^{2}-2 n_{2} r_{3} n_{3} r_{2}+r_{2}^{2} n_{3}^{2}+1-n_{2}^{2}-n_{3}^{2} \\
& =r_{1}^{2}-n_{2}^{2}\left(1-r_{3}^{2}\right)-2 n_{2} r_{2} n_{3} r_{3}-n_{3}^{2}\left(1-r_{2}^{2}\right)+1 \\
& =r_{1}^{2}-n_{2}^{2}\left(r_{1}^{2}+r_{2}^{2}\right)-2 n_{2} r_{2} n_{3} r_{3}-n_{3}^{2}\left(r_{1}^{2}+r_{3}^{2}\right)+1 \\
& =r_{1}^{2}\left(1-n_{2}^{2}-n_{3}^{2}\right)-\left(n_{2} r_{2}+n_{3} r_{3}\right)^{2}+1 \\
& =r_{1}^{2} n_{1}^{2}-\left(-n_{1} r_{1}\right)^{2}+1=1 .
\end{aligned}
$$

\section{A Quadratic Equation}

Theorem 1: Let $\boldsymbol{n}$ be the unit normal vector of the plane and let vectors $\boldsymbol{r}$ and $\boldsymbol{s}$ satisfy $(\boldsymbol{r}, \boldsymbol{r})=1,(\boldsymbol{n}, \boldsymbol{r})=0$, $\boldsymbol{s}=\boldsymbol{n} \times \boldsymbol{r}$ and condition (7). Putting

$$
\begin{aligned}
& \beta_{1}=\left(D_{+} \boldsymbol{r}, D_{-} \boldsymbol{r}\right)=\frac{r_{1}^{2}}{a^{2}}+\frac{r_{2}^{2}}{b^{2}}-\frac{r_{3}^{2}}{c^{2}}, \\
& \beta_{2}=\left(D_{+} \boldsymbol{s}, D_{-} \boldsymbol{s}\right)=\frac{s_{1}^{2}}{a^{2}}+\frac{s_{2}^{2}}{b^{2}}-\frac{s_{3}^{2}}{c^{2}},
\end{aligned}
$$

$\beta_{1}$ and $\beta_{2}$ are solutions of the following quadratic Equation:

$$
\begin{aligned}
& \beta^{2}-\left[n_{1}^{2}\left(\frac{1}{b^{2}}-\frac{1}{c^{2}}\right)+n_{2}^{2}\left(\frac{1}{a^{2}}-\frac{1}{c^{2}}\right)+n_{3}^{2}\left(\frac{1}{a^{2}}+\frac{1}{b^{2}}\right)\right] \beta \\
& -\frac{n_{1}^{2}}{b^{2} c^{2}}-\frac{n_{2}^{2}}{a^{2} c^{2}}+\frac{n_{3}^{2}}{a^{2} b^{2}}=0 .
\end{aligned}
$$

Proof: Utilising Corollary 1 one obtains:

$$
\begin{aligned}
\beta_{1}+\beta_{2} & =\frac{r_{1}^{2}+s_{1}^{2}}{a^{2}}+\frac{r_{2}^{2}+s_{2}^{2}}{b^{2}}-\frac{r_{3}^{2}+s_{3}^{2}}{c^{2}} \\
& =\frac{1-n_{1}^{2}}{a^{2}}+\frac{1-n_{2}^{2}}{b^{2}}-\frac{1-n_{3}^{2}}{c^{2}} \\
& =\frac{n_{2}^{2}+n_{3}^{2}}{a^{2}}+\frac{n_{1}^{2}+n_{3}^{2}}{b^{2}}-\frac{n_{1}^{2}+n_{2}^{2}}{c^{2}} \\
& =n_{1}^{2}\left(\frac{1}{b^{2}}-\frac{1}{c^{2}}\right)+n_{2}^{2}\left(\frac{1}{a^{2}}-\frac{1}{c^{2}}\right)+n_{3}^{2}\left(\frac{1}{a^{2}}+\frac{1}{b^{2}}\right) .
\end{aligned}
$$

Applying diagonality condition (7) and the identity of Lagrange (16) leads to:

$$
\begin{aligned}
\beta_{1} \beta_{2} & =\left(D_{+} \boldsymbol{r}, D_{-} \boldsymbol{r}\right)\left(D_{+} \boldsymbol{s}, D_{-} \boldsymbol{s}\right) \\
& =\left(D_{+} \boldsymbol{r}, D_{-} \boldsymbol{r}\right)\left(D_{+} \boldsymbol{s}, D_{-} \boldsymbol{s}\right)-\left(D_{+} \boldsymbol{r}, D_{-} \boldsymbol{s}\right)^{2} \\
& =\left(D_{+} \boldsymbol{r} \times D_{+} \boldsymbol{s}, D_{-} \boldsymbol{r} \times D_{-} \boldsymbol{s}\right) .
\end{aligned}
$$

For the cross products $D_{ \pm} \boldsymbol{r} \times D_{ \pm} \boldsymbol{s}$ one obtains:

$$
\begin{aligned}
D_{ \pm} \boldsymbol{r} \times D_{ \pm} \boldsymbol{s} & =\left|\begin{array}{ccc}
e_{1} & e_{2} & e_{3} \\
\frac{r_{1}}{a} & \frac{r_{2}}{b} & \pm \frac{r_{3}}{c} \\
\frac{s_{1}}{a} & \frac{s_{2}}{b} & \pm \frac{s_{3}}{c}
\end{array}\right|=\left(\begin{array}{c} 
\pm \frac{r_{2} s_{3}-s_{2} r_{3}}{b c} \\
\frac{s_{1} r_{3}-r_{1} s_{3}}{a c} \\
\frac{r_{1} s_{2}-s_{1} r_{2}}{a b}
\end{array}\right) \\
& =\tilde{D}_{ \pm}(\boldsymbol{r} \times \boldsymbol{s})
\end{aligned}
$$

with the diagonal matrices

$$
\tilde{D}_{ \pm}=\operatorname{diag}\left( \pm \frac{1}{b c}, \pm \frac{1}{a c}, \frac{1}{a b}\right) .
$$

According to Grassmann's expansion theorem for the double cross product (17)

$$
\begin{aligned}
\tilde{D}_{ \pm}(\boldsymbol{r} \times \boldsymbol{s}) & =\tilde{D}_{ \pm}(\boldsymbol{r} \times(\boldsymbol{n} \times \boldsymbol{r})) \\
& =\tilde{D}_{ \pm}((\boldsymbol{r}, \boldsymbol{r}) \boldsymbol{n}-(\boldsymbol{r}, \boldsymbol{n}) \boldsymbol{r})=\tilde{D}_{ \pm} \boldsymbol{n}
\end{aligned}
$$

follows, since $(\boldsymbol{r}, \boldsymbol{r})=1$ and $(\boldsymbol{r}, \boldsymbol{n})=0$. Applying (20), (21), (23) one obtains:

$$
\beta_{1} \beta_{2}=\left(\tilde{D}_{+} \boldsymbol{n}, \tilde{D}_{-} \boldsymbol{n}\right)=-\frac{n_{1}^{2}}{b^{2} c^{2}}-\frac{n_{2}^{2}}{a^{2} c^{2}}+\frac{n_{3}^{2}}{a^{2} b^{2}} .
$$

Corollary 2: Under the assumptions of Theorem 1 the following three pairs of Equations are valid:

$$
\begin{aligned}
& D_{ \pm} \boldsymbol{r} \times D_{ \pm} \boldsymbol{s}=\tilde{D}_{ \pm}(\boldsymbol{r} \times \boldsymbol{s})=\tilde{D}_{ \pm} \boldsymbol{n}, \\
& D_{ \pm} \boldsymbol{n} \times D_{ \pm} \boldsymbol{r}=\tilde{D}_{ \pm}(\boldsymbol{n} \times \boldsymbol{r})=\tilde{D}_{ \pm} \boldsymbol{s}, \\
& D_{ \pm} \boldsymbol{s} \times D_{ \pm} \boldsymbol{n}=\tilde{D}_{ \pm}(\boldsymbol{s} \times \boldsymbol{n})=\tilde{D}_{ \pm} \boldsymbol{r} .
\end{aligned}
$$

The first pair of Equations was verified in the proof of Theorem 1. The second and the third pair of Equations follow analogously.

\section{A Formular for d}

Theorem 2: Under the assumptions of Theorem 1 with $\beta_{1} \neq 0$ and $\beta_{2} \neq 0$ the expression for $d$ in (15) is given by:

$$
d=\frac{\kappa^{2}}{a^{2} n_{1}^{2}+b^{2} n_{2}^{2}-c^{2} n_{3}^{2}},
$$

where $\kappa$ is taken from (14).

Proof: The verification of (25) consists of three steps.

Step 1: Applying the identity of Lagrange (16) the following statements hold:

$$
\begin{aligned}
& \left(D_{+} \boldsymbol{n}, D_{-} \boldsymbol{n}\right)\left(D_{+} \boldsymbol{r}, D_{-} \boldsymbol{r}\right)-\left(D_{+} \boldsymbol{n}, D_{-} \boldsymbol{r}\right)^{2} \\
& =\left(D_{+} \boldsymbol{n} \times D_{+} \boldsymbol{r}, D_{-} \boldsymbol{n} \times D_{-} \boldsymbol{r}\right), \\
& \left(D_{+} \boldsymbol{n}, D_{-} \boldsymbol{n}\right)\left(D_{+} \boldsymbol{s}, D_{-} \boldsymbol{s}\right)-\left(D_{+} \boldsymbol{n}, D_{-} \boldsymbol{s}\right)^{2} \\
& =\left(D_{+} \boldsymbol{n} \times D_{+} \boldsymbol{s}, D_{-} \boldsymbol{n} \times D_{-} \boldsymbol{s}\right) .
\end{aligned}
$$


With Corollary 2 and the diagonal matrices

$$
D^{ \pm}=\operatorname{diag}(a, b, \pm c)
$$

one obtains:

$$
\begin{aligned}
& \left(D_{+} \boldsymbol{n} \times D_{+} \boldsymbol{r}, D_{-} \boldsymbol{n} \times D_{-} \boldsymbol{r}\right) \\
& =\left(\tilde{D}_{+} \boldsymbol{s}, \tilde{D}_{-} \boldsymbol{s}\right)=-\frac{\left(D^{+} \boldsymbol{s}, D^{-} \boldsymbol{s}\right)}{a^{2} b^{2} c^{2}}, \\
& \left(D_{+} \boldsymbol{n} \times D_{+} \boldsymbol{s}, D_{-} \boldsymbol{n} \times D_{-} \boldsymbol{s}\right) \\
& =\left(\tilde{D}_{+} \boldsymbol{r}, \tilde{D}_{-} \boldsymbol{r}\right)=-\frac{\left(D^{+} \boldsymbol{r}, D^{-} \boldsymbol{r}\right)}{a^{2} b^{2} c^{2}}
\end{aligned}
$$

and it follows by substituting (28) into (26)

$$
\begin{aligned}
\left(D_{+} \boldsymbol{n}, D_{-} \boldsymbol{r}\right)^{2}= & \left(D_{+} \boldsymbol{n}, D_{-} \boldsymbol{n}\right)\left(D_{+} \boldsymbol{r}, D_{-} \boldsymbol{r}\right) \\
& +\frac{\left(D^{+} \boldsymbol{s}, D^{-} \boldsymbol{s}\right)}{a^{2} b^{2} c^{2}}, \\
\left(D_{+} \boldsymbol{n}, D_{-} \boldsymbol{s}\right)^{2} & =\left(D_{+} \boldsymbol{n}, D_{-} \boldsymbol{n}\right)\left(D_{+} \boldsymbol{s}, D_{-} \boldsymbol{s}\right) \\
& +\frac{\left(D^{+} \boldsymbol{r}, D^{-} \boldsymbol{r}\right)}{a^{2} b^{2} c^{2}} .
\end{aligned}
$$

Introducing expressions

$$
\begin{aligned}
& \gamma_{1}=\left(D^{+} \boldsymbol{r}, D^{-} \boldsymbol{r}\right)=a^{2} r_{1}^{2}+b^{2} r_{2}^{2}-c^{2} r_{3}^{2}, \\
& \gamma_{2}=\left(D^{+} \boldsymbol{s}, D^{-} \boldsymbol{s}\right)=a^{2} s_{1}^{2}+b^{2} s_{2}^{2}-c^{2} s_{3}^{2},
\end{aligned}
$$

one obtains from (29) using (18) and (30)

$$
\begin{aligned}
& \left(D_{+} \boldsymbol{n}, D_{-} \boldsymbol{r}\right)^{2}=\left(D_{+} \boldsymbol{n}, D_{-} \boldsymbol{n}\right) \beta_{1}+\frac{\gamma_{2}}{a^{2} b^{2} c^{2}}, \\
& \left(D_{+} \boldsymbol{n}, D_{-} \boldsymbol{s}\right)^{2}=\left(D_{+} \boldsymbol{n}, D_{-} \boldsymbol{n}\right) \beta_{2}+\frac{\gamma_{1}}{a^{2} b^{2} c^{2}} .
\end{aligned}
$$

Combining both Equations (31) for $\beta_{1} \neq 0$ and $\beta_{2} \neq 0$ leads to

$$
\begin{aligned}
& \frac{\left(D_{+} \boldsymbol{n}, D_{-} \boldsymbol{r}\right)^{2}}{\left(D_{+} \boldsymbol{r}, D_{-} \boldsymbol{r}\right)}+\frac{\left(D_{+} \boldsymbol{n}, D_{-} \boldsymbol{s}\right)^{2}}{\left(D_{+} \boldsymbol{s}, D_{-} \boldsymbol{s}\right)} \\
& =2\left(D_{+} \boldsymbol{n}, D_{-} \boldsymbol{n}\right)+\frac{\gamma_{1} \beta_{1}+\gamma_{2} \beta_{2}}{a^{2} b^{2} c^{2} \beta_{1} \beta_{2}} .
\end{aligned}
$$

Step 2: Analogously to the verification of (24) the application of the identity of Lagrange (16) yields:

$$
\begin{aligned}
& \gamma_{1} \gamma_{2}=\left(D^{+} \boldsymbol{r}, D^{-} \boldsymbol{r}\right)\left(D^{+} \boldsymbol{s}, D^{-} \boldsymbol{s}\right) \\
& =\left(D^{+} \boldsymbol{r}, D^{-} \boldsymbol{r}\right)\left(D^{+} \boldsymbol{s}, D^{-} \boldsymbol{s}\right)-\left(D^{+} \boldsymbol{r}, D^{-} \boldsymbol{s}\right)^{2}+\left(D^{+} \boldsymbol{r}, D^{-} \boldsymbol{s}\right)^{2} \\
& =\left(D^{+} \boldsymbol{r} \times D^{+} \boldsymbol{s}, D^{-} \boldsymbol{r} \times D^{-} \boldsymbol{s}\right)+\left(D^{+} \boldsymbol{r}, D^{-} \boldsymbol{s}\right)^{2} .
\end{aligned}
$$

With the diagonal matrices

$$
\tilde{D}^{ \pm}=\operatorname{diag}( \pm b c, \pm a c, a b)
$$

for the cross products $D^{ \pm} \boldsymbol{r} \times D^{ \pm} \boldsymbol{s}$ holds:

$$
D^{ \pm} \boldsymbol{r} \times D^{ \pm} \boldsymbol{s}=\tilde{D}^{ \pm}(\boldsymbol{r} \times \boldsymbol{s})=\tilde{D}^{ \pm} \boldsymbol{n} .
$$

Therefore one obtains

$$
\gamma_{1} \gamma_{2}=\left(\tilde{D}^{+} \boldsymbol{n}, \tilde{D}^{-} \boldsymbol{n}\right)+\left(D^{+} \boldsymbol{r}, D^{-} \boldsymbol{s}\right)^{2}
$$

or

$$
\gamma_{1} \gamma_{2}=-a^{2} b^{2} c^{2}\left(D_{+} \boldsymbol{n}, D_{-} \boldsymbol{n}\right)+\left(D^{+} \boldsymbol{r}, D^{-} \boldsymbol{s}\right)^{2} .
$$

In contrast to the verification of (24), where diagonality condition (7) holds, the analogous expression $\left(D^{+} \boldsymbol{r}, D^{-} s\right)$ in (33) need not be zero.

Step 3: Applying the identity of Lagrange (16) again leads to

$$
\begin{aligned}
\left(D_{+} \boldsymbol{n} \times D_{+} \boldsymbol{r}, D_{-} \boldsymbol{n} \times D_{-} \boldsymbol{s}\right) & =\left(D_{+} \boldsymbol{n}, D_{-} \boldsymbol{n}\right)\left(D_{+} \boldsymbol{r}, D_{-} \boldsymbol{s}\right) \\
& -\left(D_{+} \boldsymbol{n}, D_{-} \boldsymbol{s}\right)\left(D_{+} \boldsymbol{r}, D_{-} \boldsymbol{n}\right) .
\end{aligned}
$$

Substituting the involved cross products according to Corollary 2 and considering diagonality condition (7) one obtains

$$
\left(\tilde{D}_{+} \boldsymbol{s}, \tilde{D}_{-} \boldsymbol{r}\right)=\left(D_{+} \boldsymbol{n}, D_{-} \boldsymbol{s}\right)\left(D_{+} \boldsymbol{n}, D_{-} \boldsymbol{r}\right),
$$

or

$$
-\frac{\left(D^{+} \boldsymbol{s}, D^{-} \boldsymbol{r}\right)}{a^{2} b^{2} c^{2}}=\left(D_{+} \boldsymbol{n}, D_{-} \boldsymbol{s}\right)\left(D_{+} \boldsymbol{n}, D_{-} \boldsymbol{r}\right) .
$$

Squaring both sides of (34) and substituting the expressions from (31) leads to:

$$
\begin{aligned}
\left(D^{+} \boldsymbol{s}, D^{-} \boldsymbol{r}\right)^{2} & =\left(a^{2} b^{2} c^{2}\left(D_{+} \boldsymbol{n}, D_{-} \boldsymbol{n}\right) \beta_{2}+\gamma_{1}\right) \\
& \cdot\left(a^{2} b^{2} c^{2}\left(D_{+} \boldsymbol{n}, D_{-} \boldsymbol{n}\right) \beta_{1}+\gamma_{2}\right) \\
& =a^{4} b^{4} c^{4}\left(D_{+} \boldsymbol{n}, D_{-} \boldsymbol{n}\right)^{2} \beta_{1} \beta_{2} \\
& +\left(\gamma_{1} \beta_{1}+\gamma_{2} \beta_{2}\right) a^{2} b^{2} c^{2}\left(D_{+} \boldsymbol{n}, D_{-} \boldsymbol{n}\right)+\gamma_{1} \gamma_{2} .
\end{aligned}
$$

Substitution of (33) results in Equation

$$
\begin{aligned}
& a^{2} b^{2} c^{2}\left(D_{+} \boldsymbol{n}, D_{-} \boldsymbol{n}\right) \\
& \cdot\left[a^{2} b^{2} c^{2}\left(D_{+} \boldsymbol{n}, D_{-} \boldsymbol{n}\right) \beta_{1} \beta_{2}+\left(\gamma_{1} \beta_{1}+\gamma_{2} \beta_{2}\right)-1\right]=0,
\end{aligned}
$$

or

$$
\gamma_{1} \beta_{1}+\gamma_{2} \beta_{2}=1-a^{2} b^{2} c^{2}\left(D_{+} \boldsymbol{n}, D_{-} \boldsymbol{n}\right) \beta_{1} \beta_{2} .
$$

Substitution of (35) in (32) leads to:

$$
\begin{aligned}
& \frac{\left(D_{+} \boldsymbol{n}, D_{-} \boldsymbol{r}\right)^{2}}{\left(D_{+} \boldsymbol{r}, D_{-} \boldsymbol{r}\right)}+\frac{\left(D_{+} \boldsymbol{n}, D_{-} \boldsymbol{s}\right)^{2}}{\left(D_{+} \boldsymbol{s}, D_{-} \boldsymbol{s}\right)} \\
& =\left(D_{+} \boldsymbol{n}, D_{-} \boldsymbol{n}\right)+\frac{1}{a^{2} b^{2} c^{2} \beta_{1} \beta_{2}} .
\end{aligned}
$$

Because of (24)

$$
a^{2} b^{2} c^{2} \beta_{1} \beta_{2}=-\left(a^{2} n_{1}^{2}+b^{2} n_{2}^{2}-c^{2} n_{3}^{2}\right)
$$

holds and with (15) one finally obtains relation (25) 


$$
\begin{aligned}
d & =\kappa^{2}\left(\left(D_{+} \boldsymbol{n}, D_{-} \boldsymbol{n}\right)-\frac{\left(D_{+} \boldsymbol{n}, D_{-} \boldsymbol{r}\right)^{2}}{\left(D_{+} \boldsymbol{r}, D_{-} \boldsymbol{r}\right)}-\frac{\left(D_{+} \boldsymbol{n}, D_{-} \boldsymbol{s}\right)^{2}}{\left(D_{+} \boldsymbol{s}, D_{-} \boldsymbol{s}\right)}\right) \\
& =\frac{\kappa^{2}}{a^{2} n_{1}^{2}+b^{2} n_{2}^{2}-c^{2} n_{3}^{2}} .
\end{aligned}
$$

Corollary 3: Under the assumptions of Theorem 1 and in case of a hyperboloid of one sheet assuming $\beta_{i}>0$ for $i=1,2$, in case of a hyperboloid of two sheets assuming $\beta_{i}>0$ for $i=1,2$ and $d<-1$, the intersection of hyperboloid (1) and a plane with unit normal vector $n$ and distance $\kappa$ from the origin is an ellipse, the area $F$ of which is given by:

$$
F=\pi\left( \pm 1+\frac{\kappa^{2}}{\kappa_{t}^{2}}\right) \frac{a b c}{\kappa_{t}} \text { with } \kappa_{t}=\sqrt{-a^{2} n_{1}^{2}-b^{2} n_{2}^{2}+c^{2} n_{3}^{2}} .
$$

In this formula +1 corresponds to a hyperboloid of one sheet, -1 to a hyperboloid of two sheets.

Proof: With $\beta_{i}>0$ for $i=1,2$ both sides of Equation (37) are positive. Thus $d$ according to (25) is negative for $\kappa \neq 0$, and zero for $\kappa=0$. In case of a hyperboloid of one sheet the numerator $1-d$ of $\alpha_{i}$ for $i=1,2$ in (12) is positive. In case of a hyperboloid of two sheets the numerator $-1-d$ of $\alpha_{i}$ for $i=1,2$ in (12) is positive for $d<-1$. Substituting $\beta_{i}$ for $i=1,2$ according to (18), $\alpha_{i}$ for $i=1,2$ are positive. In both of these cases therefore the curve of intersection (11) is an ellipse with the semi axes

$$
A=\sqrt{\frac{ \pm 1-d}{\beta_{1}}} \text { and } B=\sqrt{\frac{ \pm 1-d}{\beta_{2}}} .
$$

The area of the ellipse is given by:

$$
F=\pi A B=\pi \sqrt{\frac{ \pm 1-d}{\beta_{1}}} \sqrt{\frac{ \pm 1-d}{\beta_{2}}}=\pi \frac{ \pm 1-d}{\sqrt{\beta_{1} \beta_{2}}}
$$

By applying (25) and (37) one obtains the formula in Corollary 3.

Remark 1: In the special case that the plane of intersection of the hyperboloid is parallel to the $\mathrm{x}-\mathrm{y}$-plane, i.e. the normal vector $\boldsymbol{n}=(0,0,1)^{\mathrm{T}}$ with $\boldsymbol{\kappa}=(\boldsymbol{q}, \boldsymbol{n})=q_{3}$ and furthermore $\boldsymbol{r}=(1,0,0)^{\mathrm{T}}, \quad \boldsymbol{s}=(0,1,0)^{\mathrm{T}} \quad$ can be chosen satisfying (4), (5), (6), (7) and $\beta_{1}=\frac{1}{a^{2}}, \beta_{2}=\frac{1}{b^{2}}$, the formula for the area of the ellipse of intersection reduces to:

$$
F=\pi\left( \pm 1+\frac{q_{3}^{2}}{c^{2}}\right) a b .
$$

The same result is obtained from (1) putting $x_{3}=q_{3}$ and calculating the area of an ellipse with the semi axes

$$
a \sqrt{ \pm 1+\frac{q_{3}^{2}}{c^{2}}} \text { and } b \sqrt{ \pm 1+\frac{q_{3}^{2}}{c^{2}}} .
$$

As stated above in case of a hyperboloid of two sheets $d=-\frac{q_{3}^{2}}{c^{2}}<-1$ has to be assumed.

Remark 2: Assuming $\beta_{i}<0$ for $\mathrm{i}=1,2$ and $d>-1$ in case of a hyperboloid of two sheets would also result in positive $\alpha_{i}$ for $i=1,2$ according to (18) and (12). However for two vectors $\boldsymbol{r}$ and $\boldsymbol{s}$ in $\boldsymbol{R}^{3}$ the conditions $\beta_{i}<0$ for $i=1,2$ and $\left(D_{+} \boldsymbol{r}, D_{-} \boldsymbol{s}\right)=0$ cannot be fulfilled simultaneously.

$\beta_{i}<0$ for $i=1,2$ would imply

$$
\frac{r_{1}^{2}}{a^{2}}+\frac{r_{2}^{2}}{b^{2}}<\frac{r_{3}^{2}}{c^{2}}, \frac{s_{1}^{2}}{a^{2}}+\frac{s_{2}^{2}}{b^{2}}<\frac{s_{3}^{2}}{c^{2}},
$$

and thus

$$
\left(\frac{r_{1}^{2}}{a^{2}}+\frac{r_{2}^{2}}{b^{2}}\right)\left(\frac{s_{1}^{2}}{a^{2}}+\frac{s_{2}^{2}}{b^{2}}\right)<\frac{r_{3}^{2}}{c^{2}} \frac{s_{3}^{2}}{c^{2}} .
$$

Because of $\left(D_{+} \boldsymbol{r}, D_{-} \boldsymbol{s}\right)=0$

$$
\frac{r_{1} s_{1}}{a^{2}}+\frac{r_{2} s_{2}}{b^{2}}=\frac{r_{3} s_{3}}{c^{2}}
$$

holds. Substituting this Equation into the above inequality gives

$$
\left(\frac{r_{1}^{2}}{a^{2}}+\frac{r_{2}^{2}}{b^{2}}\right)\left(\frac{s_{1}^{2}}{a^{2}}+\frac{s_{2}^{2}}{b^{2}}\right)<\left(\frac{r_{1} s_{1}}{a^{2}}+\frac{r_{2} s_{2}}{b^{2}}\right)^{2} .
$$

Deleting equal terms on both sides of the inequality finally results in

$$
\frac{\left(r_{1} s_{2}-r_{2} s_{1}\right)^{2}}{a^{2} b^{2}}<0
$$

which is impossible for vectors $\boldsymbol{r}$ and $\boldsymbol{s}$ with real components.

Corollary 4: Under the assumptions of Theorem 1 and assuming $\beta_{1}>0$ and $\beta_{2}<0$ the intersection of hyperboloid (1) and a plane with unit normal vector $\boldsymbol{n}$ and distance $\kappa$ from the origin is for $d \neq \pm 1$ a hyperbola and for $d= \pm 1$ a pair of straight lines.

Proof: With $\beta_{1}>0$ and $\beta_{2}<0$ both sides of Equation (37) are negative. Thus $d$ according to (25) is positive or zero. In case $1-d>0$ holds for a hyperboloid of one sheet with the semi axes

$$
A=\sqrt{\frac{1-d}{\beta_{1}}} \text { and } B=\sqrt{\frac{1-d}{-\beta_{2}}} .
$$

the line of intersection is a hyperbola of the form

$$
\frac{\left(t-t_{0}\right)^{2}}{A^{2}}-\frac{\left(u-u_{0}\right)^{2}}{B^{2}}=1 \text {. }
$$


In case $1-d<0$ holds for a hyperboloid of one sheet with the semi axes

$$
A=\sqrt{\frac{-(1-d)}{\beta_{1}}} \text { and } B=\sqrt{\frac{-(1-d)}{-\beta_{2}}} .
$$

the line of intersection is a hyperbola of the form

$$
-\frac{\left(t-t_{0}\right)^{2}}{A^{2}}+\frac{\left(u-u_{0}\right)^{2}}{B^{2}}=1,
$$

with the axes interchanged.

Since $d$ is positive or zero, $-1-d<0$ is fulfilled, so that for a hyperboloid of two sheets with the semi axes

$$
A=\sqrt{\frac{-(-1-d)}{\beta_{1}}} \text { and } B=\sqrt{\frac{-(-1-d)}{-\beta_{2}}} .
$$

the line of intersection is a hyperbola of the form

$$
-\frac{\left(t-t_{0}\right)^{2}}{A^{2}}+\frac{\left(u-u_{0}\right)^{2}}{B^{2}}=1,
$$

with the axes interchanged, as in the previous case.

In case of $\pm 1-d=0$ according to (8), after substituting $\beta_{1}$ and $\beta_{2}$ from (18), the line of intersection is a pair of straight lines of the form

$$
\beta_{1}\left(t-t_{0}\right)^{2}+\beta_{2}\left(u-u_{0}\right)^{2}=0
$$

or

$$
t-t_{0}= \pm \frac{\sqrt{-\beta_{2}}}{\sqrt{\beta_{1}}}\left(u-u_{0}\right) .
$$

Remark 3: For $\beta_{1}<0$ and $\beta_{2}>0$ the roles of the variables $t$ and $u$ have to be interchanged.

\section{The Center of the Conic}

Substituting $q$ according to (14) in formulars (9) for the coordinates $\left(t_{0}, u_{0}\right)$ of the center of the conic in the plane spanned by $\boldsymbol{r}$ and $\boldsymbol{s}$ one obtains using (7):

$$
\begin{aligned}
& t_{0}=-\kappa \frac{\left(D_{+} \boldsymbol{n}, D_{-} \boldsymbol{r}\right)}{\beta_{1}}-\mu, \\
& u_{0}=-\kappa \frac{\left(D_{+} \boldsymbol{n}, D_{-} \boldsymbol{s}\right)}{\beta_{2}}-v .
\end{aligned}
$$

The center $m$ of the conic in $\boldsymbol{R}^{3}$ is given by:

$$
\begin{aligned}
\boldsymbol{m} & =\boldsymbol{q}+t_{0} \boldsymbol{r}+u_{0} \boldsymbol{s}=\kappa \boldsymbol{n}+\left(t_{0}+\mu\right) \boldsymbol{r}+\left(u_{0}+v\right) \boldsymbol{s} \\
& =\kappa\left(\boldsymbol{n}-\frac{\left(D_{+} \boldsymbol{n}, D_{-} \boldsymbol{r}\right)}{\beta_{1}} \boldsymbol{r}-\frac{\left(D_{+} \boldsymbol{n}, D_{-} \boldsymbol{s}\right)}{\beta_{2}} \boldsymbol{s}\right) .
\end{aligned}
$$

Theorem 3: Let the assumptions of Theorem 1 be fulfilled with $\beta_{1} \neq 0$ and $\beta_{2} \neq 0$. For the center $\boldsymbol{m}$ of the conic of intersection in $\boldsymbol{R}^{3}$ holds:

$$
\boldsymbol{m}=\frac{\kappa}{a^{2} n_{1}^{2}+b^{2} n_{2}^{2}-c^{2} n_{3}^{2}}\left(a^{2} n_{1}, b^{2} n_{2},-c^{2} n_{3}\right)^{\mathrm{T}} .
$$

Proof: With diagonal matrices $D^{ \pm}$from (27) and $\tilde{D}_{ \pm}$from (22) utilising

$$
\frac{1}{a^{2} b^{2} c^{2}} D^{+} D^{-}=-\tilde{D}_{+} \tilde{D}_{-}
$$

and (37) one obtains a representation of $\boldsymbol{m}$ equivalent to (40):

$$
\begin{aligned}
\boldsymbol{m} & =\frac{\kappa}{a^{2} n_{1}^{2}+b^{2} n_{2}^{2}-c^{2} n_{3}^{2}} D^{+} D^{-} \boldsymbol{n} \\
& =\frac{-\kappa}{-\beta_{1} \beta_{2}} \tilde{D}_{+} \tilde{D}_{-} \boldsymbol{n}=\frac{\kappa}{\beta_{1} \beta_{2}} \tilde{D}_{-} \tilde{D}_{+} \boldsymbol{n} .
\end{aligned}
$$

It is sufficient to show that for the difference

$$
\Delta=\boldsymbol{m}-\frac{\kappa}{\beta_{1} \beta_{2}} \tilde{D}_{-} \tilde{D}_{+} \boldsymbol{n}
$$

$(\Delta, \boldsymbol{n})=(\Delta, r)=(\Delta, s)=0$ holds. Thus the coefficients in the expansion of $\boldsymbol{\Delta}$ in $\boldsymbol{R}^{3}$ with respect to the orthonormal basis $[\boldsymbol{n}, \boldsymbol{r}, \boldsymbol{s}]$ are zero, i.e., $\boldsymbol{\Delta}$ is the zero vector.

Applying representation (39) and (24) one obtains:

$$
\begin{aligned}
(\Delta, \boldsymbol{n}) & =(\boldsymbol{m}, \boldsymbol{n})-\frac{\kappa}{\beta_{1} \beta_{2}}\left(\tilde{D}_{-} \tilde{D}_{+} \boldsymbol{n}, \boldsymbol{n}\right) \\
& =\frac{\kappa}{\beta_{1} \beta_{2}}\left(\beta_{1} \beta_{2}-\left(\tilde{D}_{+} \boldsymbol{n}, \tilde{D}_{-} \boldsymbol{n}\right)\right)=0 .
\end{aligned}
$$

Furthermore one obtains:

$$
\begin{aligned}
(\Delta, \boldsymbol{r}) & =(\boldsymbol{m}, \boldsymbol{r})-\frac{\kappa}{\beta_{1} \beta_{2}}\left(\tilde{D}_{-} \tilde{D}_{+} \boldsymbol{n}, \boldsymbol{r}\right) \\
& =-\frac{\kappa}{\beta_{1} \beta_{2}}\left(\left(D_{+} \boldsymbol{n}, D_{-} \boldsymbol{r}\right)\left(D_{+} \boldsymbol{s}, D_{-} \boldsymbol{s}\right)+\left(\tilde{D}_{+} \boldsymbol{n}, \tilde{D}_{-} \boldsymbol{r}\right)\right)
\end{aligned}
$$

and by interchanging the roles of $r$ and $s$ :

$$
\begin{aligned}
(\Delta, \boldsymbol{s}) & =(\boldsymbol{m}, \boldsymbol{s})-\frac{\kappa}{\beta_{1} \beta_{2}}\left(\tilde{D}_{-} \tilde{D}_{+} \boldsymbol{n}, \boldsymbol{s}\right) \\
& =-\frac{\kappa}{\beta_{1} \beta_{2}}\left(\left(D_{+} \boldsymbol{n}, D_{-} \boldsymbol{s}\right)\left(D_{+} \boldsymbol{r}, D_{-} \boldsymbol{r}\right)+\left(\tilde{D}_{+} \boldsymbol{n}, \tilde{D}_{-} \boldsymbol{s}\right)\right) .
\end{aligned}
$$

Both previous expressions are zero; this follows by applying diagonality condition (7), the identity of Lagrange (16) and Corollary 2:

$$
\begin{aligned}
& \left(D_{+} \boldsymbol{n}, D_{-} \boldsymbol{r}\right)\left(D_{+} \boldsymbol{s}, D_{-} \boldsymbol{s}\right) \\
& =\left(D_{+} \boldsymbol{n}, D_{-} \boldsymbol{r}\right)\left(D_{+} \boldsymbol{s}, D_{-} \boldsymbol{s}\right)-\left(D_{+} \boldsymbol{r}, D_{-} \boldsymbol{s}\right)\left(D_{+} \boldsymbol{s}, D_{-} \boldsymbol{n}\right) \\
& =\left(D_{+} \boldsymbol{r} \times D_{+} \boldsymbol{s}, D_{-} \boldsymbol{n} \times D_{-} \boldsymbol{s}\right)=-\left(\tilde{D}_{+} \boldsymbol{n}, \tilde{D}_{-} \boldsymbol{r}\right) .
\end{aligned}
$$

Interchanging the roles of $\boldsymbol{r}$ and $\boldsymbol{s}$ leads to:

$$
\begin{aligned}
& \left(D_{+} \boldsymbol{n}, D_{-} \boldsymbol{s}\right)\left(D_{+} \boldsymbol{r}, D_{-} \boldsymbol{r}\right) \\
& =\left(D_{+} \boldsymbol{n}, D_{-} \boldsymbol{s}\right)\left(D_{+} \boldsymbol{r}, D_{-} \boldsymbol{r}\right)-\left(D_{+} \boldsymbol{s}, D_{-} \boldsymbol{r}\right)\left(D_{+} \boldsymbol{r}, D_{-} \boldsymbol{n}\right) \\
& =\left(D_{+} \boldsymbol{s} \times D_{+} \boldsymbol{r}, D_{-} \boldsymbol{n} \times D_{-} \boldsymbol{r}\right)=-\left(\tilde{D}_{+} \boldsymbol{n}, \tilde{D}_{-} \boldsymbol{s}\right) .
\end{aligned}
$$


Corollary 5: Under the same assumptions as in Corollary 3 the line of intersection of hyperboloid (1) and a plane is an ellipse with the semi axes $A$ and $B$, given in the proof of Corollary 3, and the apexes

$$
\boldsymbol{m} \pm A \boldsymbol{r} \text { and } \boldsymbol{m} \pm B \boldsymbol{s} .
$$

Proof: Clearly $\boldsymbol{m} \pm A \boldsymbol{r}$ and $\boldsymbol{m} \pm B \boldsymbol{s}$ are points of the plane cutting the hyperboloid. In order to show that they are belonging to the ellipse of intersection, it has to be verified that they are situated on hyperboloid (1), i.e. the following equalities hold:

$$
\begin{aligned}
& \left(D_{+}(\boldsymbol{m} \pm A \boldsymbol{r}), D_{-}(\boldsymbol{m} \pm A \boldsymbol{r})\right)= \pm 1 \\
& \left(D_{+}(\boldsymbol{m} \pm B \boldsymbol{s}), D_{-}(\boldsymbol{m} \pm B \boldsymbol{s})\right)= \pm 1 .
\end{aligned}
$$

This can be verified using $\boldsymbol{m}$ in the form (39) and employing condition (7) and Equation (15).

Corollary 6: Under the same assumptions as in Corollary 4 the line of intersection of hyperboloid (1) and a plane is in case of $d \neq \pm 1$ a hyperbola with the semi axes $A$ and $B$ given in the proof of Corollary 4 . The center of the hyperbola given in (9) is equal to the point of intersection of the asymptotes of the hyperbola.

Proof: The asymptotes of the hyperbola are given by

$$
\frac{\left(t-t_{0}\right)^{2}}{A^{2}}-\frac{\left(u-u_{0}\right)^{2}}{B^{2}}=0
$$

with

$$
A=\sqrt{\frac{| \pm 1-d|}{\beta_{1}}} \text { and } B=\sqrt{\frac{| \pm 1-d|}{-\beta_{2}}}
$$

or

$$
t-t_{0}= \pm \frac{\sqrt{-\beta_{2}}}{\sqrt{\beta_{1}}}\left(u-u_{0}\right) .
$$

The point of intersection of the asymptotes $\left(t_{s}, u_{s}\right)$ fulfills the following linear system

$$
\begin{aligned}
& \left(t_{s}-t_{0}\right)-\sqrt{\frac{-\beta_{2}}{\beta_{1}}}\left(u_{s}-u_{0}\right)=0 \\
& \left(t_{s}-t_{0}\right)+\sqrt{\frac{-\beta_{2}}{\beta_{1}}}\left(u_{S}-u_{0}\right)=0 .
\end{aligned}
$$

As this homogeneous linear system for the unknowns $t_{S}-t_{0}$ and $u_{S}-u_{0}$ has a nonzero determinant, it can only have the trivial solution, which implies

$$
\left(t_{s}, u_{S}\right)=\left(t_{0}, u_{0}\right)=\left(-\frac{\left(D_{+} \boldsymbol{q}, D_{-} \boldsymbol{r}\right)}{\beta_{1}},-\frac{\left(D_{+} \boldsymbol{q}, D_{-} s\right)}{\beta_{2}}\right) .
$$

\section{Corollary 7:}

$$
\left(D_{+} \boldsymbol{m}, D_{-} \boldsymbol{m}\right)=d
$$

Proof: This can be verified, as in the proof of Corollary 5, using $\boldsymbol{m}$ in the form (39) and employing condition (7) and Equation (15).

Because of Corollary 7

$d<1$ holds, if and only if $\boldsymbol{m}$ is an interior point of a hyperboloid of one sheet,

$d<-1$ holds, if and only if $\boldsymbol{m}$ is an interior point of a hyperboloid of two sheets,

$d>1$ holds, if and only if $\boldsymbol{m}$ is an exterior point of a hyperboloid of one sheet,

$d>-1$ holds, if and only if $\boldsymbol{m}$ is an exterior point of a hyperboloid of two sheets.

In case of $d= \pm 1$ one obtains from (25)

$$
\kappa^{2}= \pm\left(a^{2} n_{1}^{2}+b^{2} n_{2}^{2}-c^{2} n_{3}^{2}\right) .
$$

The center (40) of the conic of intersection therefore becomes a tangent contact point

$$
\boldsymbol{m}=\boldsymbol{m}_{t}= \pm \frac{1}{\kappa}\left(a^{2} n_{1}, b^{2} n_{2},-c^{2} n_{3}\right)^{\mathrm{T}}
$$

of hyperboloid and plane, where the + -sign corresponds to a hyperboloid of one sheet and the --sign to a hyperboloid of two sheets.

Example: Determine the line of intersection of hyperboloid (1) and a plane, having the normal vector $(i, j, k)^{\mathrm{T}}$ and containing the point $\boldsymbol{q}=\left(q_{1}, q_{2}, q_{3}\right)^{\mathrm{T}}$, situated in the interior or on the boundary of (1):

$$
\left(x-q_{1}\right) i+\left(y-q_{2}\right) j+\left(z-q_{3}\right) k=0 .
$$

The unit normal vector of the plane has the form:

$$
\boldsymbol{n}=\frac{1}{\sqrt{i^{2}+j^{2}+k^{2}}}(i, j, k)^{\mathrm{T}} .
$$

The distance of the plane from the origin is given by:

$$
\kappa=(\boldsymbol{q}, \boldsymbol{n})=\frac{q_{1} i+q_{2} j+q_{3} k}{\sqrt{i^{2}+j^{2}+k^{2}}} .
$$

According to (25) $d$ can be written as:

$$
d=\frac{\left(q_{1} i+q_{2} j+q_{3} k\right)^{2}}{a^{2} i^{2}+b^{2} j^{2}-c^{2} k^{2}} .
$$

Substituting (18) into (12) the expressions of $\alpha_{1}$ and $\alpha_{2}$ are given by

$$
\alpha_{1}=\frac{ \pm 1-d}{\beta_{1}} \text { and } \alpha_{2}=\frac{ \pm 1-d}{\beta_{2}},
$$

where $\beta_{1}, \beta_{2}$, satisfying $\beta_{1} \neq 0$ and $\beta_{2} \neq 0$, are solutions of Equation (19) after substituting vector $\boldsymbol{n}$ from (42):

$$
\begin{aligned}
& \left(i^{2}+j^{2}+k^{2}\right) \beta^{2} \\
& -\left[i^{2}\left(\frac{1}{b^{2}}-\frac{1}{c^{2}}\right)+j^{2}\left(\frac{1}{a^{2}}-\frac{1}{c^{2}}\right)+k^{2}\left(\frac{1}{a^{2}}+\frac{1}{b^{2}}\right)\right] \beta \\
& -\frac{i^{2}}{b^{2} c^{2}}-\frac{j^{2}}{a^{2} c^{2}}+\frac{k^{2}}{a^{2} b^{2}}=0 .
\end{aligned}
$$


With Theorem 3 one obtains by substituting $\boldsymbol{n}$ from (42) and $\kappa$ from (43) the formular for the center $\boldsymbol{m}$ of the conic given by:

$$
\boldsymbol{m}=\frac{q_{1} i+q_{2} j+q_{3} k}{a^{2} i^{2}+b^{2} j^{2}-c^{2} k^{2}}\left(a^{2} i, b^{2} j,-c^{2} k\right)^{\mathrm{T}} .
$$

In the special case of a plane containing the origin, i.e. $\boldsymbol{q}$ is the zero vector, it follows by (43), (44) and (47) that $\kappa=0, d=0$ and $\boldsymbol{m}$ is the zero vector also. Furthermore the expressions of $\alpha_{1}$ and $\alpha_{2}$ in (45) reduce to

$$
\alpha_{1}=\frac{ \pm 1}{\beta_{1}} \text { and } \alpha_{2}=\frac{ \pm 1}{\beta_{2}} \text {. }
$$

As described in Corollary 3 for a hyperboloid of one sheet and $\beta_{i}>0$ for $i=1,2$ one obtains $\alpha_{i}>0$ for $i=1,2$. Then the line of intersection is an ellipse. As stated in Corollary 4 for a hyperboloid of one sheet and $\beta_{1}>0, \beta_{2}<0$ one obtains $\alpha_{1}>0, \alpha_{2}<0$. For a hyperboloid of two sheets and $\beta_{1}>0, \beta_{2}<0$ one obtains $\alpha_{1}<0, \alpha_{2}>0$. In both of these cases the line of intersection is a hyperbola.

In a second special case with $\boldsymbol{q}=(i, j, k)^{\mathrm{T}}$. the above formulas (43), (44) and (47) reduce to:

$$
\kappa=\sqrt{i^{2}+j^{2}+k^{2}}, \quad d=\frac{\left(i^{2}+j^{2}+k^{2}\right)^{2}}{a^{2} i^{2}+b^{2} j^{2}-c^{2} k^{2}}
$$

and

$$
\boldsymbol{m}=\frac{i^{2}+j^{2}+k^{2}}{a^{2} i^{2}+b^{2} j^{2}-c^{2} k^{2}}\left(a^{2} i, b^{2} j,-c^{2} k\right)^{\mathrm{T}} .
$$

Because of $\boldsymbol{q}=\kappa \boldsymbol{n}$ in (14) $\mu=v=0$ holds and (38) reduces to

$$
t_{0}=-\kappa \frac{\left(D_{+} \boldsymbol{n}, D_{-} \boldsymbol{r}\right)}{\beta_{1}}, \quad u_{0}=-\kappa \frac{\left(D_{+} \boldsymbol{n}, D_{-} \boldsymbol{s}\right)}{\beta_{2}},
$$

where $\beta_{1}$ and $\beta_{2}$ are solutions of the quadratic Equation (46) and vectors $\boldsymbol{r}$ and $\boldsymbol{s}$ have to be determined as described above in Section 2. As stated in Corollaries 3 and 4 , if $\alpha_{i}$ for $i=1,2$ are both positive, an ellipse as curve of intersection is obtained, and if $\alpha_{i}$ for $i=1,2$ are of different sign, a hyperbola as curve of intersection results.

\section{Parabola as Curve of Intersection}

A parabola (13) as curve of intersection is obtained in case of $\beta_{1}=\left(D_{+} \boldsymbol{r}, D_{-} \boldsymbol{r}\right)=0$ and $\left(D_{+} \boldsymbol{q}, D_{-} \boldsymbol{r}\right) \neq 0$. A hyperboloid of one sheet, given in (1), may be factorized in the following form:

$$
\left(\frac{x_{1}}{a}+\frac{x_{3}}{c}\right)\left(\frac{x_{1}}{a}-\frac{x_{3}}{c}\right)=\left(1+\frac{x_{2}}{b}\right)\left(1-\frac{x_{2}}{b}\right)
$$

With the decomposition

$$
\begin{aligned}
& \frac{x_{1}}{a}+\frac{x_{3}}{c}=k\left(1+\frac{x_{2}}{b}\right), \\
& \frac{x_{1}}{a}-\frac{x_{3}}{c}=\frac{1}{k}\left(1-\frac{x_{2}}{b}\right)
\end{aligned}
$$

for any value of $k \in \boldsymbol{R}, k \neq 0$, these Equations represent a straight line, as the intersection of two planes in $\boldsymbol{R}^{3}$. This straight line lies on (48) because, if the members of (49) are multiplied together, (48) results. Rearranging (49) one obtains

$$
\begin{aligned}
& \frac{x_{1}}{a}-k \frac{x_{2}}{b}+\frac{x_{3}}{c}-k=0, \\
& \frac{x_{1}}{a}+\frac{1}{k} \frac{x_{2}}{b}-\frac{x_{3}}{c}-\frac{1}{k}=0 .
\end{aligned}
$$

With the abbreviations

$$
l=\frac{k^{2}-1}{k b c}, m=\frac{2}{a c}, n=\frac{1+k^{2}}{k a b}
$$

the straigth line (50) can be equivalently rewritten [3]

$$
x_{1}=p_{1}+l v, x_{2}=p_{2}+m v, x_{3}=p_{3}+n v
$$

with a point $\boldsymbol{p}=\left(p_{1}, p_{2}, p_{3}\right)^{\mathrm{T}}$ on (50) and $v \in \boldsymbol{R}$.

Putting

$$
\boldsymbol{r}=\frac{1}{\sqrt{l^{2}+m^{2}+n^{2}}}(l, m, n)^{\mathrm{T}}
$$

$$
\begin{aligned}
\beta_{1}=( & \left.D_{+} \boldsymbol{r}, D_{-} \boldsymbol{r}\right)=0 \text { holds, because } \\
& \beta_{1}\left(l^{2}+m^{2}+n^{2}\right)=\left(\frac{l^{2}}{a^{2}}+\frac{m^{2}}{b^{2}}-\frac{n^{2}}{c^{2}}\right) \\
= & \left(\frac{\left(k^{2}-1\right)^{2}}{k^{2} a^{2} b^{2} c^{2}}+\frac{4}{a^{2} b^{2} c^{2}}-\frac{\left(1+k^{2}\right)^{2}}{k^{2} a^{2} b^{2} c^{2}}\right)=0 .
\end{aligned}
$$

Choosing a vector $\boldsymbol{q}=\left(q_{1}, q_{2}, q_{3}\right)^{\mathrm{T}}$ on the surface of a hyperboloid of one sheet, as given in (1), for instance

$$
\begin{aligned}
& \boldsymbol{q}=(a \sin \varphi, b \cos \varphi, 0)^{T} \quad \text { with } \varphi \in[0,2 \pi), \\
& \left(D_{+} \boldsymbol{q}, D_{-} \boldsymbol{r}\right) \sqrt{l^{2}+m^{2}+n^{2}}=\left(\sin \varphi \frac{l}{a}+\cos \varphi \frac{m}{b}\right) \\
& =\left(\sin \varphi \frac{k^{2}-1}{k a b c}+\cos \varphi \frac{2}{a b c}\right) \neq 0 \\
& \text { for } \varphi=0, \frac{\pi}{2}, \pi, \frac{3 \pi}{2} \text { and } k \neq \pm 1 .
\end{aligned}
$$

results.

Constructing a vector $s$, fulfilling

$$
(\boldsymbol{r}, \boldsymbol{s})=0, \quad\left(D_{+} r, D_{-} s\right)=0, \quad(\boldsymbol{s}, \boldsymbol{s})=1,
$$

a plane spanned by vectors $\boldsymbol{r}$ and $\boldsymbol{s}$ is obtained, con- 
taining the straight line (52). The two linear Equations in (54) for the components of $s$ can be rewritten:

$$
\begin{aligned}
& l s_{1}+m s_{2}=-n s_{3} \\
& l \frac{s_{1}}{a^{2}}+m \frac{s_{2}}{b^{2}}=n \frac{s_{3}}{c^{2}} .
\end{aligned}
$$

Solving for $s_{1}$ and $s_{2}$ under the assumptions $k \neq \pm 1$ and $a \neq b$ gives:

$$
\begin{aligned}
& s_{1}=-\frac{n}{l} \frac{a^{2}\left(c^{2}+b^{2}\right)}{c^{2}\left(a^{2}-b^{2}\right)} s_{3} \\
& s_{2}=\frac{n}{m} \frac{b^{2}\left(c^{2}+a^{2}\right)}{c^{2}\left(a^{2}-b^{2}\right)} s_{3} .
\end{aligned}
$$

Dividing by $s_{3} \neq 0$ one obtains $\sigma_{i}=\frac{s_{i}}{s_{3}}$ for $i=1,2$ and thus the following normalized vector $s$ :

$$
\boldsymbol{s}=\frac{1}{\sqrt{\sigma_{1}^{2}+\sigma_{2}^{2}+1}}\left(\sigma_{1}, \sigma_{2}, 1\right)^{\mathrm{T}}
$$

fulfilling (54) and giving

$$
\beta_{2}=\left(D_{+} s, D_{-} s\right)=\frac{1}{\sigma_{1}^{2}+\sigma_{2}^{2}+1}\left(\frac{\sigma_{1}^{2}}{a^{2}}+\frac{\sigma_{2}^{2}}{b^{2}}-\frac{1}{c^{2}}\right) .
$$

In case $a=b$, this signifies rotational symmetry of the hyperboloid with regard to the $\mathrm{z}$-axis, the coefficient matrix of (55) is singular. The condition for solvability of (55) is

$$
\operatorname{Rg}\left(\begin{array}{cc}
l & m \\
\frac{l}{a^{2}} & \frac{m}{b^{2}}
\end{array}\right)=\operatorname{Rg}\left(\begin{array}{ccc}
l & m & -n s_{3} \\
\frac{l}{a^{2}} & \frac{m}{b^{2}} & \frac{n}{c^{2}} s_{3}
\end{array}\right) .
$$

As $a=b$ this can be reduced to

$$
R g\left(\begin{array}{cc}
l & m \\
0 & 0
\end{array}\right)=R g\left(\begin{array}{ccc}
l & m & -n s_{3} \\
0 & 0 & \left(\frac{n}{c^{2}}+\frac{n}{a^{2}}\right) s_{3}
\end{array}\right) .
$$

Both sides of (56) are equal to 1 only for $s_{3}=0$. A solution vector $s$ may then be chosen as

$$
\boldsymbol{s}=\frac{1}{\sqrt{m^{2}+l^{2}}}(m,-l, 0)^{\mathrm{T}}
$$

fulfilling (54). This leads to

$$
\beta_{2}=\left(D_{+} s, D_{-} s\right)=\frac{1}{m^{2}+l^{2}}\left(\frac{m^{2}}{a^{2}}+\frac{l^{2}}{a^{2}}\right)=\frac{1}{a^{2}} .
$$

For $k= \pm 1$ according to (51) $l=0$ results. Then the linear system (55) is solvable for arbitrary $S_{1}$ and $s_{2}=s_{3}=0$. Choosing $s=(1,0,0)^{\mathrm{T}}$, as above $\beta_{2}=\frac{1}{a^{2}}$ holds.
Using vector $\boldsymbol{q}$ given in (53)

$$
\left(D_{+} \boldsymbol{q}, D_{-} \boldsymbol{q}\right)=\frac{a^{2} \sin ^{2} \varphi}{a^{2}}+\frac{b^{2} \cos ^{2} \varphi}{b^{2}}=1
$$

is obtained. Thus parabola (13) has the form

$$
2\left(D_{+} \boldsymbol{q}, D_{-} \boldsymbol{r}\right) t+\beta_{2}\left(u-u_{0}\right)^{2}=\frac{\left(D_{+} \boldsymbol{q}, D_{-} \boldsymbol{s}\right)^{2}}{\beta_{2}}
$$

with

$$
u_{0}=-\frac{\left(D_{+} \boldsymbol{q}, D_{-} \boldsymbol{s}\right)}{\beta_{2}} .
$$

Instead of (49) the alternative decomposition of (48)

$$
\begin{aligned}
& \frac{x_{1}}{a}+\frac{x_{3}}{c}=\tilde{k}\left(1-\frac{x_{2}}{b}\right), \\
& \frac{x_{1}}{a}-\frac{x_{3}}{c}=\frac{1}{\tilde{k}}\left(1+\frac{x_{2}}{b}\right)
\end{aligned}
$$

for any value of $\tilde{k} \in \boldsymbol{R}, \tilde{k} \neq 0$, may be considered; (58) also describes a straight line as intersection of two planes in $\boldsymbol{R}^{3}$. This straight line as well lies on (48) because, if the members of (58) are multiplied together, (48) results. Rearranging (58) one obtains

$$
\begin{aligned}
& \frac{x_{1}}{a}+\tilde{k} \frac{x_{2}}{b}+\frac{x_{3}}{c}-\tilde{k}=0, \\
& \frac{x_{1}}{a}-\frac{1}{\tilde{k}} \frac{x_{2}}{b}-\frac{x_{3}}{c}-\frac{1}{\tilde{k}}=0 .
\end{aligned}
$$

With the abbreviations

$$
\tilde{l}=-\frac{\tilde{k}^{2}-1}{\tilde{k} b c}, \tilde{m}=\frac{2}{a c}, \tilde{n}=-\frac{1+\tilde{k}^{2}}{\tilde{k} a b} .
$$

the straigth line (59) can be equivalently rewritten [3]

$$
x_{1}=p_{1}+\tilde{l} v, x_{2}=p_{2}+\tilde{m} v, x_{3}=p_{3}+\tilde{n} v
$$

with a point $\boldsymbol{p}=\left(p_{1}, p_{2}, p_{3}\right)^{\mathrm{T}}$ on (59) and $v \in \boldsymbol{R}$.

As previously with the terms $l, m, n$ now with the terms $\tilde{l}, \tilde{m}, \tilde{n}$ vectors $\boldsymbol{r}$ and $\boldsymbol{s}$ can be defined satisfying

$$
\begin{aligned}
& \beta_{1}=\left(D_{+} \boldsymbol{r}, D_{-} \boldsymbol{r}\right)=0, \\
& (\boldsymbol{r}, \boldsymbol{s})=0, \quad\left(D_{+} \boldsymbol{r}, D_{-} \boldsymbol{s}\right)=0, \quad(\boldsymbol{s}, \boldsymbol{s})=1 .
\end{aligned}
$$

Choosing a vector $\boldsymbol{q}$ as in (53), in the end a parabola of the form (57) is obtained.

Mathematica programs modelling the cases described in Corollaries 3 and 4 and in Section 6 may be obtained from the author upon request.

\section{Conclusion}

The intention of this paper is to look at cases which are not treated in mathematical textbooks where the plane 
intersecting a hyperboloid of one sheet or of two sheets is not necessarily parallel to the coordinate planes and thus produces all kinds of conics: ellipses, hyperbolas and parabolas.

\section{REFERENCES}

[1] P. P. Klein, "On the Ellipsoid and Plane Intersection Equation," Applied Mathematics, Vol. 3, No. 11, 2012, pp.
1634-1640. http://dx.doi.org/10.4236/am.2012.311226

[2] A. Korn and M. Korn, "Mathematical Handbook for Scientists and Engineers," Mc Graw-Hill Book Company, Inc., New York, Toronto, London, 1961.

[3] I. N. Bronshtein, K. A. Semendyayev, G. Musiol, H. Muehlig, "Handbook of Mathematics," 5th Edition, Springer, Berlin, Heidelberg, New York, 2007. 